\title{
AQP8 and AQP9 expression in patients with polycystic ovary syndrome and its association with in vitro fertilization-embryo transfer outcomes
}

\author{
ZHENGFANG XIONG ${ }^{1,2}$, BING LI $^{3}$, LIYUN WANG $^{2}$, XIANGHUI ZENG ${ }^{2}$, \\ BINYE $\mathrm{LI}^{2}$, XIAOYAN SHA ${ }^{4}$ and HUISHU LIU ${ }^{4}$
}

\author{
${ }^{1}$ Department of Gynaecology and Obstetrics, The First Affiliated Hospital of Jinan University, \\ Guangzhou, Guangdong 510623; Departments of ${ }^{2}$ Reproductive Medical Center and ${ }^{3}$ General Surgery, \\ Qinghai Provincial People's Hospital, Xining, Qinghai 810000; ${ }^{4}$ Department of Gynecology and Obstetrics, Guangzhou \\ Women and Children's Medical Center, Guangzhou Medical University, Guangzhou, Guangdong 510623, P.R. China
}

Received May 23,2018; Accepted March 7, 2019

DOI: $10.3892 / \mathrm{etm} .2019 .7592$

\begin{abstract}
The aim of the present study was to investigate aquaporin (AQP)8 and AQP9 expression in patients with polycystic ovary syndrome (PCOS) and its association with in vitro fertilization-embryo transfer (IVF-ET) outcomes. A total of 45 patients with PCOS undergoing IVF-ET (test group) and 50 patients with oviduct obstruction or ovarian cyst (control group) were assessed for the mRNA expression of AQP8 and AQP9 in ovarian tissues by reverse transcription-quantitative (RT-q)PCR. The levels of luteinizing hormone, anti-mullerian hormone and testosterone were determined, which were revealed to be significantly different between the two groups $(\mathrm{P}<0.05)$. The RT-qPCR results indicated that AQP8 expression in the control group was lower than that in the test group $(\mathrm{t}=37.75, \mathrm{P}<0.01)$, whereas AQP9 expression in the control group was higher than that in the test group $(\mathrm{t}=19.59, \mathrm{P}<0.01)$. The number of eggs obtained in the group with high AQP8 expression was significantly lower than that in the group with low AQP8 expression ( $\mathrm{t}=2.64, \mathrm{P}<0.01)$. The number of high-quality embryos in the high AQP8 expression group was not significantly different from that in the low AQP8 expression group ( $\mathrm{t}=1.02, \mathrm{P}>0.05)$. The pregnancy rate in patients with high AQP9 expression was higher than that in the low AQP9 expression group $(\mathrm{P}<0.05)$ and the abortion rate in the former was lower than that in the latter $(\mathrm{P}<0.05)$. In conclusion, AQP8 and AQP9 are differentially expressed in ovarian tissues of patients with PCOS vs. normal control subjects. The
\end{abstract}

Correspondence to: Dr Huishu Liu, Department of Gynecology and Obstetrics, Guangzhou Women and Children's Medical Center, Guangzhou Medical University, 9 Jinsui Road, Guangzhou, Guangdong 510623, P.R. China

E-mail: huishuliu@hotmail.com

Key words: aquaporin 8, aquaporin 9, polycystic ovary syndrome, in vitro fertilization-embryo transfer expression of AQP8 is closely associated with the occurrence and development of oocytes, whereas the expression of AQP9 is associated with the success rate of pregnancy in patients with PCOS.

\section{Introduction}

Polycystic ovary syndrome (PCOS) is a common but complex reproductive dysfunction and is an endocrine disorder comprising an abnormal glucose metabolism (1). Surveys have revealed that PCOS is mainly detected in adolescent females and women of childbearing age, and the incidence rate is $\sim 10 \%$ of all women globally (2). Other studies have indicated that $>70 \%$ of female cases of infertility and $\sim 40 \%$ of spontaneous abortions are linked to PCOS (3). PCOS is associated with sparse ovulation or prolonged anovulation and significantly elevated androgen levels, while its clinical manifestations have a high degree of heterogeneity amongst patients (4).

In the early stage of the disease, infertility, obesity and menstrual disorders may occur. In the advanced stage, the risk of cardiovascular and cerebrovascular diseases and type II diabetes increases significantly, which markedly affects the quality of life, as well as the physical and mental health of patients (5). At present, the majority of studies suggest that PCOS is caused by genetic mutations as well as antiepileptic drugs, particularly valproate (6). Although the in vitro fertilization-embryo transfer (IVF-ET) technique is a routine method to remedy infertility in patients with PCOS wishing to conceive, the associated endocrine and glucose metabolism disorder induces abnormal follicular development, which leads to the production of poor-quality embryos in patients with PCOS undergoing IVF-ET. This results in fewer oocytes available for the patient and reduces the success rate of the transplantation (7).

Aquaporin (AQP) is a hydrophobic internal membrane protein that selectively lets water molecules pass, which is an important pathway for water to diffuse through the cell membrane (protein-mediated water transport) apart from physical diffusion (8). To date, 13 types of AQPs, AQP0-12, 
have been discovered in mammals. AQPs are small-molecule proteins with a molecular weight of 25-34 kDa that are located on the membranes of epithelial and endothelial cells (9). The family of AQPs is divided into three subgroups based on their genetic structure: Classical aquaporin proteins (AQP0-2, -4-6 and -8), glycerol-water channel proteins (AQP3, -7, -9 and -10), and super AQP (AQP11 and -12) (10). A study suggested that AQPs are selectively expressed in the membranes of cells of the reproductive system tissue, with their functions including involvement in saliva secretion and the re-absorption and transportation of water molecules, suggesting a close association between AQP and the maintenance of the reproductive system (11). In a study by McConnell et al (12), flow cytometric analysis revealed that AQP7-9 were present on the cell membranes of rats, and these AQPs were involved in water transportation of granulosa cells and associated with the development of follicles.

Therefore, the present study examined the association between the relative expression levels of AQP8 and -9 in ovary tissues of patients with PCOS and examined their possible associations with the outcomes of IVF-ET.

\section{Materials and methods}

Reagents. The reverse transcriptase and reverse transcription (RT) kit (iScript Advanced cDNA Synthesis kit; cat. no. 1708842) was purchased from Takara Bio, Inc. The $2 \mathrm{X}$ SYBR Green quantitative PCR (qPCR) Mix and the RevertAid First Strand complementary (c)DNA Synthesis kit (DyNAmo Flash SYBR Green qPCR kit; cat. no. F415S) were purchased from Invitrogen (Thermo Fisher Scientific, Inc.), and the $\beta$-actin, AQP8 and AQP9 primers (Sangon Biotech Co., Ltd.) were designed and synthesized by Shanghai Sangon Biotech. The primer sequences used are listed in Table I.

Clinical information of patients. A total of 45 cases with PCOS undergoing IVF-ET (test group) at Guangzhou Women and Children's Medical Center (Guangdong, China) from January 2015 to May 2016 were included in the present study. They had an average age of $28.45 \pm 4.86$ years with an age range of 24 to 35 years. The patients were diagnosed based on the assessment criteria of the American Society for Reproductive Medicine and the European Society of Human Reproduction and Embryology $(13,14)$. In the control group, 50 cases were included that had oviduct obstruction or ovarian cysts and who had undergone ovary biopsies with an age range of 25-34 years and an average age of $28.21 \pm 4.23$ years. The inclusion criteria included sparse or prolonged anovulation or irregular ovulation (determined by blood and ovulation tests), elevated hormone expression levels, increased ovarian size, and $>12$ follicles on each side (diameter, 2-8 mm). The exclusion criteria were a family history of disease (e.g., diabetes), endocrine disorders (e.g., congenital adrenal hyperplasia, hypothyroidism or hyperthyroidism and hyperprolactinemia), no compliance with the treatment and follow-up schedule and spouses with infertility. The present study was approved by the Ethics Committee of the First Affiliated Hospital of Jinan University (Guangzhou, China), and the patients and their families provided written informed consent.
Medication protocol. The superovulation regimen (standard long-term ovulation promotion) included Inda (ethinyl estradiol cyproterone tablets) administered orally, 1 tablet per day on the third day of the first week of the menstrual period. The long-acting Daphneline $(1.25 \mathrm{mg}$ ) was intramuscularly injected on the 17th day of the administration of Inda and patients were treated with Gonafin (recombinant follicle-stimulating hormone; $150 \mathrm{U} /$ day) by intramuscular injection to promote follicular growth when the adjustment was satisfactory. When $>2$ follicle diameters measuring $18 \mathrm{~mm}$ and $>3$ measuring $17 \mathrm{~mm}$ were reached, human chorionic gonadotropin (HCG; $10,000 \mathrm{U}$ ) was intramuscularly injected and the eggs were retrieved after 35-36 h. The IVF/intracytoplasmic sperm injection was performed based on standard clinical practice and at three days after oocyte retrieval, the oocytes were selected. A total of 2-3 embryos were selected for transplantation and, from the remaining oocytes, 2-4 high-quality embryos were preserved in liquid nitrogen at $-126^{\circ} \mathrm{C}$. The low-quality oocytes were further cultured for 5-6 days and the embryos formed were reserved in liquid nitrogen at $-126^{\circ} \mathrm{C}$ for subsequent use if a high-quality embryo was unavailable.

Pregnancy outcomes. The patients who had received IVF for 14 days were tested for fasting progesterone and HCG hormone expression levels. If the expression level of HCG was $\geq 5 \mathrm{IU} / \mathrm{ml}$ and, after a further 2 weeks, the gestational sac within the uterus was observed by B-ultrasound, clinical pregnancy was diagnosed.

Identification of high-quality embryos. Embryos were divided into four grades: Level I, uniform blastomeres and a cytoplasmic fraction of $<10 \%$; level II, slightly uneven blastomeres and cytoplasmic fraction of 10-20\%; level III, slightly uneven blastomeres and cytoplasmic fraction of $21-50 \%$; and grade IV, cytoplasmic fraction of $>50 \%$. Embryos with $>6$ cells and a grade of I/II were rated as high-quality embryos.

Specimen collection. Ovary tissues were obtained after resection from the fallopian tube (e.g., laparoscopic ovarian wedge resection) for patients in the control group or the ovarian tissue was obtained by ovarian biopsy during surgery for ovarian cysts.

Detection of relative expression of AQP8 and AQP9 $\mathrm{mRNA}$ in ovarian tissues by $R T$-qPCR. The ovarian tissues were repeatedly ground with a grinding rod and shaken with TRIzol reagent (Thermo Fisher Scientific, Inc.), and lysed for $30 \mathrm{~min}$. The purity and concentration of the extracted total RNA was determined by UV spectrophotometry. The ratio of the optical density at 260 vs. $280 \mathrm{~nm}$ was within the range of 1.8-2.1. Complementary (c)DNA was synthesized by strictly following the manufacturer's protocols for AQP8 and AQP9 mRNA. The PCR system contained $100 \mathrm{ng} / \mu \mathrm{l} \mathrm{cDNA}$ template, $0.5 \mathrm{~mol} / \mathrm{l}$ of the primer, $2.0 \mu \mathrm{l}$ of $2 \mathrm{X} \mathrm{dNTP}, 2.5 \mu \mathrm{l}$ of the buffer solution, $1.5 \mathrm{~mol} / \mathrm{l}$ of $\mathrm{MgCl}_{2}$ and $1.0 \mathrm{IU}$ of Taq DNA polymerase, which was adjusted to a total volume of $20 \mu \mathrm{l}$ using nuclease-free water. PCR conditions were as follows: Pre-denaturation at $95^{\circ} \mathrm{C}$ for $5 \mathrm{~min}, 95^{\circ} \mathrm{C}$ for $30 \mathrm{sec}, 60^{\circ} \mathrm{C}$ for $15 \mathrm{sec}$ and $60^{\circ} \mathrm{C}$ for $15 \mathrm{sec}$, for a total of 45 cycles. U6 was used as the reference gene and the $2^{-\triangle \Delta \mathrm{Cq}}$ method (15) was applied to determine the expression level. 
Table I. Sequences of primers used for PCR.

\begin{tabular}{lll}
\hline Gene & \multicolumn{1}{c}{ Upstream primer } & \multicolumn{1}{c}{ Downstream primer } \\
\hline AQP8 & 5'-CGGATGTCTATCGGTCATTGAGAA-3' & 5'-GCGACACAGCAGGGTTGAAG-3' \\
AQP9 & 5'-GGTCTTGAAGAGCAGCTTAG-3' & 5'-GTTCGCCAGAGATAGATACG-3' \\
$\beta$-actin & 5'-TTCCAGCCTTCCTTCTGG-3' & 5'-TTGCGCTCAGGAGGAGCAAT-3'
\end{tabular}

AQP, aquaporin.

Statistical analysis. SPSS 22.0 statistical software (IBM Corp.) was used to analyze the data and GraphPad Prism 5 (GraphPad Inc.) was used to generate the figures. Measurement data are expressed as the mean \pm standard deviation. The comparison between groups was based on the normality test results from the t-test. An independent t-test was used for comparison between groups. A paired t-test was used for comparisons between the before and after group and a rank sum test was used for data that did not conform to normal distribution. Count data were expressed as $\mathrm{n}(\%)$ and comparisons between groups were performed using the Chi-squared test. $\mathrm{P}<0.05$ was considered to indicate a statistically significant difference.

\section{Results}

Clinical data of the two groups of patients. When comparing the clinical information between the two groups of patients, no significant difference in any of the indicators was identified ( $P>0.05$; Table II), except for luteinizing hormone, anti-mullerian hormone and testosterone levels $(\mathrm{P}<0.05)$.

AQP8 and AQP9 $m R N A$ expression in the two groups of patients. AQP8 and AQP9 mRNA expression in the ovarian tissues of the two groups of patients was detected by RT-qPCR. The levels of AQP8 mRNA in the control group were significantly lower than those in the test group and there was a significant difference between the two groups $(\mathrm{t}=37.75, \mathrm{P}<0.01$; Fig. 1A). The AQP9 mRNA expression levels in the control group were significantly higher than those in the test group and there was a statistically significant difference between the two groups ( $\mathrm{t}=19.59, \mathrm{P}<0.01$; Fig. 1B).

Embryonic quality and number of oocytes retrieved from the test group. Based on the median expression levels of AQP8 and AQP9 mRNA, the patients were divided into high expression and low expression groups. The quality of embryos and the number of oocytes obtained were compared between these groups, and it was indicated that the number of oocytes obtained in the AQP8 mRNA high expression group was significantly lower than that obtained in the low expression group $(\mathrm{t}=2.64$, $\mathrm{P}<0.01$ ); however, there was no significant difference in the number of high-quality embryos between the AQP8 mRNA high and low expression groups $(\mathrm{t}=1.02, \mathrm{P}>0.05)$. There was no significant difference in the number of oocytes obtained from patients with high vs. low AQP9 mRNA expression $(\mathrm{t}=0.71$, $\mathrm{P}>0.05$ ), and there was no significant difference in the number of high-quality embryos between the high and low expression groups of AQP9 mRNA ( $\mathrm{t}=1.01, \mathrm{P}>0.05$; Table III).
Observation of pregnancy outcomes of patients. Patients were grouped based on the median expression levels of AQP8 and AQP9 mRNA. The pregnancy rate in the AQP8 mRNA high expression group was not significantly different from that in the AQP8 mRNA low expression group $(\mathrm{P}>0.05)$. There was no significant difference in the rate of abortions between the two groups $(\mathrm{P}>0.05)$. The pregnancy rate among patients in the high AQP9 mRNA expression group was significantly higher than that in the AQP9 mRNA low expression group $(\mathrm{P}<0.05)$. The high AQP9 mRNA expression group also had a lower miscarriage rate than the AQP9 mRNA low expression group $(\mathrm{P}<0.05$; Table IV).

\section{Discussion}

As a gynecological endocrine disease, PCOS has a complex range of causes, which include the metabolic system, endocrine system and ovarian regulation system (16). Patients with PCOS encountered in the clinic may also have varying types and degrees of metabolic disorders (e.g., insulin resistance, high blood lipids and high blood pressure); however, what impairs the reproductive health of females the most is ovulation dysfunction. The mechanisms of ovulation abnormalities are highly complex and may be induced by factors including hypothalamic-pituitary-ovarian function disorders and abnormal hormone feedback signals in the body (17).

As one of the gene families that have been receiving an increased amount of attention from researchers in recent years, AQPs constitute an important route of water transportation that is separate from simple diffusion (18). Previous studies have indicated that AQPs have a regulatory role in functions associated with the mammalian reproductive system and affect the formation of the embryo sac; they may cause changes in the uterine cavity fluid at the implantation stage and the formation of the oviduct (19). A difference in the transportation of substances via AQP8 has been noted among different species, and it has been indicated that AQP8 facilitates water and urea transportation in mice, but fails to transport glycerol, whereas it transports only water in rats but not urea and glycerol (20). However, in recent years, the known roles of AQP8 have expanded, i.e., in addition to the expression on the cell membrane, expression of AQP8 has also been observed on the mitochondrial membrane (21). The extensive expression of AQP8 in various tissue types in the body has been assessed numerous times and it has been indicated that AQP8 mRNA expression is present in ovarian tissues. As one of the members of the AQP family, AQP9 is highly expressed in the liver, and varying degrees 
Table II. Comparison of clinicopathological and demographic characteristics between the two groups of subjects [n(\%)].

\begin{tabular}{|c|c|c|c|c|}
\hline Parameter & Observation group $(n=45)$ & Control group $(\mathrm{n}=50)$ & $\mathrm{t} / \chi^{2}$ & P-value \\
\hline Duration of infertility (years) & & & 0.01 & 0.91 \\
\hline$>2 \mathrm{n}(\%)$ & $23(51.11)$ & $25(52.08)$ & & \\
\hline$\leq 2 \mathrm{n}(\%)$ & $22(46.81)$ & $25(53.19)$ & & \\
\hline Age (years) & & & 0.01 & 0.96 \\
\hline$>30 \mathrm{n}(\%)$ & $25(55.56)$ & $28(56.00)$ & & \\
\hline$\leq 30 \mathrm{n}(\%)$ & $20(44.44)$ & $22(44.00)$ & & \\
\hline Degree of education & & & 0.22 & 0.63 \\
\hline$<$ High school n (\%) & $15(33.33)$ & $19(38.00)$ & & \\
\hline$\geq$ High school n (\%) & $30(66.67)$ & $31(62.00)$ & & \\
\hline Smoking history & & & 0.33 & 0.56 \\
\hline Yes n $(\%)$ & $3(6.67)$ & $2(4.00)$ & & \\
\hline No n $(\%)$ & $42(93.33)$ & $48(96.00)$ & & \\
\hline History of alcoholism & & & 0.33 & 0.56 \\
\hline Yes n $(\%)$ & $3(6.67)$ & $2(4.00)$ & & \\
\hline No n $(\%)$ & $42(93.33)$ & $48(96.00)$ & & \\
\hline Area of residence & & & 0.66 & 0.41 \\
\hline Rural area n (\%) & $11(24.44)$ & $16(32.00)$ & & \\
\hline City n (\%) & $34(75.56)$ & $34(68.00)$ & & \\
\hline Body mass index $\left(\mathrm{kg} / \mathrm{m}^{2}\right)$ & $22.54 \pm 2.62$ & $21.84 \pm 2.15$ & 1.42 & 0.15 \\
\hline FSH (IU/l) & $5.95 \pm 2.38$ & $6.49 \pm 3.29$ & 0.91 & 0.37 \\
\hline LH (IU/l) & $12.39 \pm 8.12$ & $5.92 \pm 3.88$ & 5.03 & $<0.01$ \\
\hline AMH (ng/ml) & $9.68 \pm 4.21$ & $5.37 \pm 4.97$ & 4.53 & $<0.01$ \\
\hline $\mathrm{T}(\mathrm{ng} / \mathrm{ml})$ & $0.69 \pm 0.24$ & $0.51 \pm 0.18$ & 4.16 & $<0.01$ \\
\hline
\end{tabular}

Values are expressed as $\mathrm{n}(\%)$ or the mean \pm standard deviation. FSH, follicle-stimulating hormone; LH, luteinizing hormone; AMH, anti-mullerian hormone; $\mathrm{T}$, testosterone.
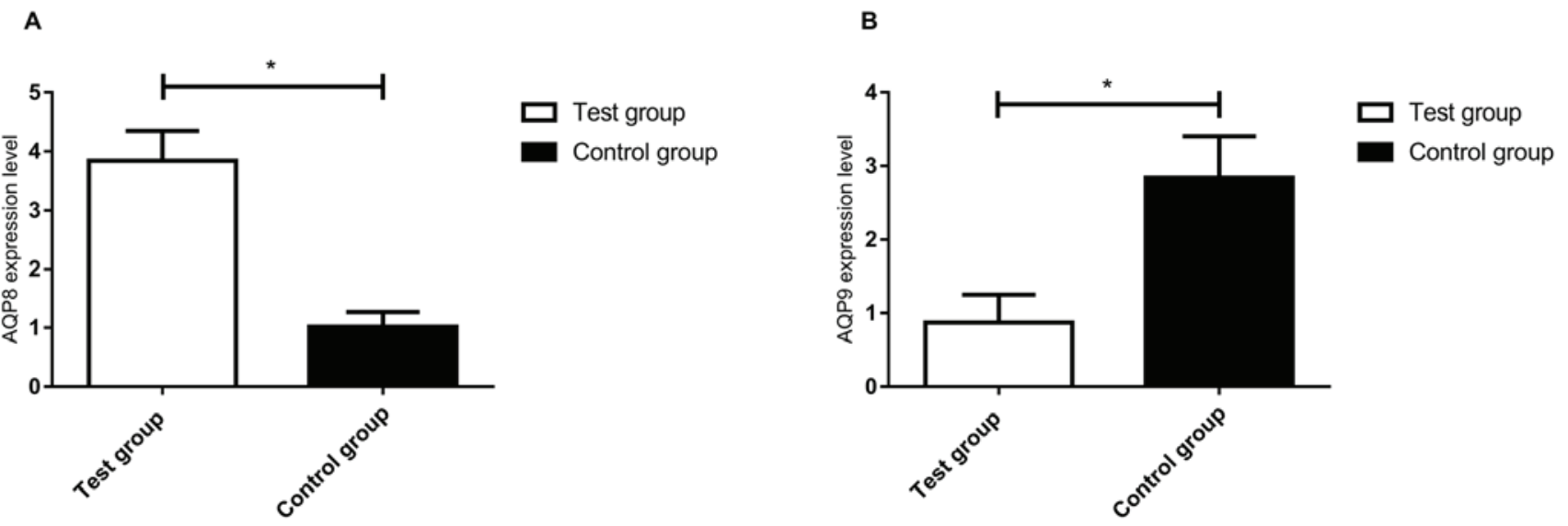

Figure 1. Expression of AQP8 mRNA in the two groups was detected by reverse transcription-quantitative polymerase chain reaction. (A) The expression of AQP8 mRNA in the control group was significantly lower than that in the test group. (B) Expression of AQP9 mRNA in the two groups was detected by reverse transcription-quantitative polymerase chain reaction. The expression of AQP9 mRNA in the control group was significantly higher than that in the test group. ${ }^{*} \mathrm{P}<0.01$. AQP, aquaporin.

of differential expression among different individuals have been reported in the testis, nervous system and thyroid system (22). In a study by Sales et al (23), AQP9 was indicated to have an important role in the development of follicular cells. However, there is a dispute on its expression in patients with PCOS. In a previous study on PCOS, no difference in AQP9 expression in ovarian tissues was identified between the test group and the control group and yet, low expression 
Table III. Embryo quality and egg count in the test group.

\begin{tabular}{|c|c|c|c|c|c|c|c|c|}
\hline \multirow[b]{2}{*}{ Group } & \multicolumn{2}{|c|}{ AQP8 } & \multirow[b]{2}{*}{$\mathrm{t}$} & \multirow[b]{2}{*}{ P-value } & \multicolumn{2}{|c|}{ AQP9 } & \multirow[b]{2}{*}{$\mathrm{t}$} & \multirow[b]{2}{*}{ P-value } \\
\hline & $\begin{array}{l}\text { High level } \\
\quad(n=17)\end{array}$ & $\begin{array}{l}\text { Low level } \\
\qquad(\mathrm{n}=28)\end{array}$ & & & $\begin{array}{l}\text { High level } \\
\quad(n=22)\end{array}$ & $\begin{array}{c}\text { Low level } \\
(n=23)\end{array}$ & & \\
\hline Oocytes retrieved (n) & $11.84 \pm 3.54$ & $14.36 \pm 2.81$ & 2.64 & 0.01 & $13.58 \pm 2.52$ & $13.08 \pm 2.17$ & 0.71 & 0.47 \\
\hline High-quality embryos (n) & $10.84 \pm 2.76$ & $9.86 \pm 3.29$ & 1.02 & 0.31 & $12.28 \pm 2.28$ & $11.48 \pm 2.94$ & 1.01 & 0.31 \\
\hline
\end{tabular}

Values are expressed as the mean \pm standard deviation. AQP, aquaporin.

Table IV. Pregnancy-associated outcomes in the test group.

\begin{tabular}{|c|c|c|c|c|c|c|}
\hline \multirow[b]{2}{*}{ Group } & \multicolumn{2}{|c|}{ AQP8 } & \multirow[b]{2}{*}{ P-value } & \multicolumn{2}{|c|}{ AQP9 } & \multirow[b]{2}{*}{ P-value } \\
\hline & High level $(\mathrm{n}=17)$ & Low level (n=28) & & High level (n=22) & Low level $(n=23)$ & \\
\hline Conception & & & 0.76 & & & 0.02 \\
\hline No & $10(58.82)$ & $18(64.29)$ & & $4(18.18)$ & $11(47.83)$ & \\
\hline Yes & $7(41.18)$ & $10(35.71)$ & & $18(81.82)$ & $12(52.17)$ & \\
\hline Spontaneous abortion & & & 0.49 & & & 0.04 \\
\hline No & $14(82.35)$ & $20(71.43)$ & & $16(72.73)$ & $9(39.13)$ & \\
\hline Yes & $3(17.65)$ & $8(28.57)$ & & $6(27.27)$ & $14(60.87)$ & \\
\hline
\end{tabular}

AQP, aquaporin. Values are expressed as $\mathrm{n}(\%)$.

was detected in the granulosa cells of the PCOS patients (24). In the present study, a significant reduction of AQP9 mRNA was detected in the ovarian tissue of PCOS patients compared to the control group. The discrepancy of these results may be due to the recruitment of more patients in the current study, using different tissues.

In the present study, AQP8 and AQP9 mRNA expression in the ovarian tissue of the two groups of patients was detected by RT-qPCR. AQP8 mRNA expression in the PCOS group was significantly higher than that in the control group, while AQP9 mRNA expression was significantly lower. In previous studies (25-27), ovarian tissue and follicular fluid were examined by PCR and it was revealed that AQP9 mRNA expression was significantly lower in the test group compared with that in the control group, suggesting that patients with high expression of AQP8 and low expression of AQP9 may have PCOS. AQP8 and AQP9 are expected to become biological indicators for the condition. A study by $\mathrm{Su}$ et al (28), indicated that in female AQP8-knockout mice, the ovulation numbers were higher than those in wild-type mice. In the present study, the PCOS patients were divided into high and low expression groups based on the median value of AQP8 mRNA expression, and the number of ovulations in the low expression group was significantly higher than that in the high expression group in patients undergoing IVF-ET. This may be due to the water transportation in granulosa cells being affected by the reduced expression of AQP8, which may result in a reduction of apoptosis among granulosa cells, as well as inhibition of follicular disappearance and atresia. This indicates that AQP8 expression is closely associated with the generation and development of egg cells. At the time of completion of the present study, no difference in the number of non-pregnant patients and the rate of abortions was noted between the AQP8 mRNA high and low expression groups. By contrast, the number of non-pregnant patients and patients with miscarriage in the AQP9 mRNA high group was significantly lower than that in the AQP9 low expression group. This proved the association between the expression of AQP9 and pregnancy-associated processes in PCOS patients.

Of note, the present study has several limitations. First, only a small number of patient samples were analyzed, which may have affected the results. Furthermore, only the ovarian tissue of PCOS patients was examined, while the follicle fluid was not analyzed; therefore, it remains elusive whether any possible difference in the follicle fluid levels may have had an influence. In addition, the underlying mechanism for the difference in AQP8 and AQP9 levels were not explored with regard to patients with PCOS. A future study will endeavor to increase the number of subjects and the type of samples analyzed, and further explore the association between AQP8 and AQP9 in PCOS to determine the accuracy of the experimental results and further confirm the validity of the conclusions.

In conclusion, a differential expression of AQP8 and AQP9 in ovarian tissues was identified between PCOS patients and normal patients. AQP8 expression was closely associated with the development of oocytes and AQP9 expression was associated with the success of pregnancy in PCOS patients. 


\section{Acknowledgements}

Not applicable.

\section{Funding}

The current study was supported by Guangdong Provincial Science and Technology Hall (grant no. 2016A020218002).

\section{Availability of data and materials}

The complete data are available from the corresponding author on reasonable request. The data are not publicly available due to their containing information that could compromise the privacy of research participants.

\section{Authors' contributions}

ZX and HL conceived the study and designed the experiments, $\mathrm{BL}, \mathrm{LW}$ and $\mathrm{XZ}$ contributed to data collection, and BYL and $\mathrm{XS}$ performed the data analysis and interpreted the results. ZX wrote the manuscript and HL contributed to the critical revision of the article. All authors read and approved the final manuscript.

\section{Ethics approval and consent to participate}

This study was approved by the ethics committee of Guangzhou Women and Children's Medical Center (Guangzhou Medical University, Guangzhou, China). Written informed consent was obtained from the patients and their families.

\section{Patient consent for publication}

Not applicable.

\section{Competing interests}

The authors declare that they have no competing interests.

\section{References}

1. Rosenblum J and Ekhlaspour L: Polycystic Ovary Syndrome. In The MassGeneral Hospital for Children Adolescent Medicine Handbook. Goldstein MA (ed). Springer, pp187-193, 2017.

2. Goetsch AL, Kimelman D and Woodruff TK: Polycystic Ovary Syndrome. In: Fertility Preservation and Restoration for Patients with Complex Medical Conditions. Springer, pp231-248, 2017.

3. Connolly A and Beckett VA (eds): Women's Health in Primary Care. Cambridge University Press, Cambridge, ppi-ii, 2017.

4. Johnston-MacAnanny EB and Berga SL: Polycystic Ovary Syndrome. In: Clinical Reproductive Medicine and Surgery. Springer, pp123-137, 2017.

5. Franks S: Polycystic ovary syndrome. N Engl J Med 333: 853-861, 1995

6. Zaki M, Hassan N, El-Bassyouni HT, Kamal S, Basha W, Azmy O and Amr K: Association of the Pro12Ala polymorphism with the metabolic parameters in women with polycystic ovary syndrome. Open Access Maced J Med Sci 5: 275-280, 2017.

7. Chen WY, Du YQ, Guan X, Zhang HY and Liu T: Effect of GnRHR polymorphisms on in vitro fertilization and embryo transfer in patients with polycystic ovary syndrome. J Hum Genet 62: 1065-1071, 2017.
8. Bienert GP and Chaumont F: Aquaporin-facilitated transmembrane diffusion of hydrogen peroxide. Biochim Biophys Acta 1840: 1596-1604, 2014.

9. Ishibashi K, Kondo S, Hara S and Morishita Y: The evolutionary aspects of aquaporin family. Am J Physiol Regul Integr Comp Physiol 300: R566-R576, 2011.

10. Zhu C, Jiang Z, Bazer FW, Johnson GA, Burghardt RC and Wu G: Aquaporins in the female reproductive system of mammals. Front Biosci (Landmark Ed) 20: 838-871, 2015

11. Mobasheri A, Marples D, Young IS, Floyd RV, Moskaluk CA and Frigeri A: Distribution of the AQP4 water channel in normal human tissues: Protein and tissue microarrays reveal expression in several new anatomical locations, including the prostate gland and seminal vesicles. Channels (Austin) 1: 29-38, 2007.

12. McConnell NA, Yunus RS, Gross SA, Bost KL, Clemens MG and Hughes FM Jr: Water permeability of an ovarian antral follicle is predominantly transcellular and mediated by aquaporins. Endocrinology 143: 2905-2912, 2002.

13. Society for Assisted Reproductive Technology; American Society for Reproductive Medicine: Assisted reproductive technology in the United States: 2000 results generated from the American Society for Reproductive Medicine/Society for Assisted Reproductive Technology Registry. Fertil Steril 81: 1207-1220, 2004.

14. European Society of Human Genetics; European Society of Human Reproduction and Embryology: The need for interaction between assisted reproduction technology and genetics: Recommendations of the European Societies of Human Genetics and Human Reproduction and Embryology. Hum Reprod 21: 1971-1973, 2006

15. Livak KJ and Schmittgen TD: Analysis of relative gene expression data using real-time quantitative PCR and the 2(-Delta Delta C(T)) method. Methods 25: 402-408, 2001.

16. Azziz R, Carmina E, Chen Z, Dunaif A, Laven JS, Legro RS, Lizneva D, Natterson-Horowtiz B, Teede HJ and Yildiz BO: Polycystic ovary syndrome. Nat Rev Dis Primers 2: 16057, 2016.

17. Gong JG, Campbell BK, Bramley TA, Gutierrez CG, Peters AR and Webb R: Suppression in the secretion of follicle-stimulating hormone and luteinizing hormone, and ovarian follicle development in heifers continuously infused with a gonadotropin-releasing hormone agonist. Biol Reprod 55: 68-74, 1996.

18. Jozefkowicz C, Scochera F and Alleva K: Two aquaporins, multiple ways of assembly. Channels (Austin) 10: 438-439, 2016.

19. Yeste M, Morató R, Rodríguez-Gil J, Bonet $\mathrm{S}$ and Prieto-Martínez N: Aquaporins in the male reproductive tract and sperm: Functional implications and cryobiology. Reprod Domest Anim 52 (Suppl 4): S12-S27, 2017.

20. Ferri D, Mazzone A, Liquori GE, Cassano G, Svelto M and Calamita G: Ontogeny, distribution, and possible functional implications of an unusual aquaporin, AQP8, in mouse liver. Hepatology 38: 947-957, 2003.

21. Saparov SM, Liu K, Agre P and Pohl P: Fast and selective ammonia transport by aquaporin-8. J Biol Chem 282: 5296-5301, 2007.

22. Elkjær M, Vajda Z, Nejsum LN, Kwon T, Jensen UB, Amiry-Moghaddam M, Frøkiaer $J$ and Nielsen S: Immunolocalization of AQP9 in liver, epididymis, testis, spleen, and brain. Biochem Biophys Res Commun 276: 1118-1128, 2000.

23. Sales A, Lobo C, Carvalho A, Moura A and Rodrigues A: Structure, function, and localization of aquaporins: Their possible implications on gamete cryopreservation. Genet Mol Res 12: 6718-6732, 2013.

24. Qu F, Wang FF, Lu XE, Dong MY, Sheng JZ, Lv PP, Ding GL, Shi BW, Zhang D and Huang HF: Altered aquaporin expression in women with polycystic ovary syndrome: Hyperandrogenism in follicular fluid inhibits aquaporin-9 in granulosa cells through the phosphatidylinositol 3-kinase pathway. Hum Reprod 25: 1441-1450, 2010.

25. Maeda N, Funahashi T and Shimomura I: Metabolic impact of adipose and hepatic glycerol channels aquaporin 7 and aquaporin 9. Nat Clin Pract Endocrinol Metab 4: 627-634, 2008.

26. Suzuki-Toyota F, Ishibashi K and Yuasa S: Immunohistochemical localization of a water channel, aquaporin 7 (AQP7), in the rat testis. Cell Tissue Res 295: 279-285, 1999.

27. Richard C, Gao J, Brown N and Reese J: Aquaporin water channel genes are differentially expressed and regulated by ovarian steroids during the periimplantation period in the mouse. Endocrinology 144: 1533-1541, 2003.

28. Su W, Guan X, Zhang D, Sun M, Yang L, Yi F, Hao F, Feng X and Ma T: Occurrence of multi-oocyte follicles in aquaporin 8-deficient mice. Reprod Biol Endocrinol 11: 88, 2013. 